\title{
Prévision de la variabilité intra- et interarbre de la densité du bois de chêne rouvre (Quercus petraea Liebl) par modélisation des largeurs et des densités des bois initial et final en fonction de l'âge cambial, de la largeur de cerne et du niveau dans l'arbre*
}

\author{
R Degron, G Nepveu** \\ Équipe de recherches sur la qualité des bois, Inra, 54280 Champenoux, France
}

(Reçu le 26 septembre 1994 ; accepté le 10 octobre 1995)

\begin{abstract}
Summary - Predicting intra- and intertree variability of wood density in sessile oak (Quercus petraea Liebl) through modelling earlywood and latewood width and density from cambial age, ring width and height in the tree. The oak wood intraring density being considered as an important criterion of its quality, we studied ring microdensitometric components variability (earlywood density, earlywood width, latewood density, latewood width). First, we considered the intratree variation of density with cambial age, ring width and level in tree. We used regression methods to establish models of variation with the variables we had considered and we studied parameters of the different models (table II) by analysis of variance. Within a tree, we showed that earlywood width could be considered as a constant (fig 1) and that earlywood and latewood density were only dependent on cambial age (fig 2). For ring width and cambial age given, the level in the tree effect was important only on the lowest level of our sampling (tables III and IV). We then studied intertree density variations on 24 trees collected in northeastern France (table I). We evaluated the site effect and the tree in site effect on the parameters of the models considered beforehand (table $V$ ). We found a 'site' effect and a 'tree in site' effect on some parameters; however, these results will have to be carefully assessed because of the relative weakness of our sampling. Nevertheless, we can confirm the important wood density variability between trees.
\end{abstract}

variability / wood / Quercus petraea / density / model

Résumé - La densité intracerne du bois de chêne étant considérée comme un critère important de sa qualité, nous avons étudié la variabilité des composantes microdensitométriques du cerne (densité du bois initial, largeur du bois initial, densité du bois final, largeur du bois final). Dans une première partie, nous nous sommes intéressés à la variabilité intra-arbre de la densité, avec l'âge cambial, la largeur de cerne et le niveau dans l'arbre. Nous avons utilisé des méthodes de régression pour établir

${ }^{*}$ Cet article a fait l'objet d'une communication au cours d'un Workshop lufro du groupe de travail S5.01.04, qui s'est déroulé en Suède, du 13 au 17 juin 1994. Le thème du congrès était “Connection between silviculture and wood quality through modelling approaches and simulation softwares ".

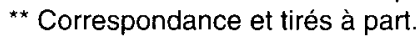


des modèles de variations des différentes variables considérées et nous avons étudié les paramètres de ces modèles par analyse de variance. Nous avons montré que dans l'arbre la largeur du bois initial pouvait être considérée constante et que la densité du bois initial et du bois final ne dépendaient que de l'âge. À largeur de cerne et âge fixés, l'effet du niveau dans l'arbre est significatif. Les paramètres observés au niveau le plus bas de notre échantillonnage sont statistiquement différents de ceux des hauteurs supérieures. Dans une seconde partie, nous avons étudié la variabilité interindividuelle de la densité du bois; nous avons travaillé sur vingt-quatre arbres provenant du nord-est de la France. Nous avons en particulier étudié l'effet du site et de l'arbre dans le site sur les paramètres des modèles mentionnés ci-dessus. Nous avons trouvé des effets " arbre dans site " et " site " sur certains des paramètres, mais ces résultats sont à considérer avec prudence compte tenu de la relative faiblesse de notre échantillonnage. Nous pouvons cependant confirmer l'importance de la variabilité interindividuelle sur la densité du bois.

\section{variabilité / bois / Quercus petraea / densité / modèle}

\section{INTRODUCTION}

Aujourd'hui, les sylviculteurs commencent à disposer de modèles de croissance des peuplements forestiers de chênes rouvres (Quercus petraea Liebl). Pour simplifier, ces modèles de croissance permettent, pour un scénario sylvicole donné appliqué sur un site de fertilité connue, de connaître la courbe donnant la largeur de cerne en fonction de l'âge depuis la moelle à tous les niveaux des arbres constituant le peuplement (De Hercé, 1992). II devient donc envisageable d'associer à ces modèles la distribution des propriétés technologiques (aptitude au tranchage, au rabotage,...) pour une meilleure prédiction globale de la qualité de cette production ligneuse. Pour ce faire, il faut identifier une ou des combinaisons de caractéristiques physiques ou anatomiques de base du bois (densité, rétractabilité, diamètre des vaisseaux,...) qui soi(en)t de bons estimateurs des propriétés technologiques et disposer de modèles de variation intra-arbre de ces caractéristiques de base afin de pouvoir connecter les modèles correspondant aux modèles de croissance.

Bien que l'on puisse envisager plusieurs caractéristiques de base pour définir la qualité du bois de chêne rouvre (Nepveu, 1990), la densité du bois est reconnue chez cette espèce comme un estimateur synthétique de la qualité de ce bois et de sa struc- ture anatomique (Polge, 1966 ; Ferrand, 1982 ; Marchal, 1983 ; Eyono Owoundi, 1991). C'est donc sur cette caractéristique physique que nous avons établi des modèles de variation intra-arbre de la qualité du bois et que nous avons étudié la variabilité entre les arbres des paramètres de ces modèles.

Nous décrirons tout d'abord le matériel de notre étude et nous montrerons les limites de notre échantillonnage. Nous présenterons ensuite notre modélisation intracerne de la densité de bois de chêne, définie sur la base d'une décomposition en bois initial et en bois final de l'accroissement annuel, en fonction de l'âge compté depuis la moelle (âge cambial), de la largeur de cerne et du niveau dans l'arbre. Nous accorderons une importance particulière à la qualité des paramètres des modèles établis ; c'est en effet par l'analyse de ces paramètres que nous étudierons l'effet du niveau dans l'arbre. Après ce point sur la variabilité intra-arbre de la densité, nous verrons comment se décompose la variabilité des paramètres des modèles de densité du bois entre les arbres en fonction des effets du site et de l'arbre dans le site.

\section{MATÉRIEL ET MÉTHODES}

Afin d'étudier la variabilité intra- et interarbre de la densité du bois, nous nous sommes appuyés sur 24 chênes rouvres provenant de 12 parcelles de 
dix forêts d'Alsace et de Lorraine. Nous disposions de deux arbres par site distants de 30 à $100 \mathrm{~m}$. Ces chênes étaient âgés de 100 à 200 ans à $1,30 \mathrm{~m}$. Une limite importante de notre échantillonnage réside dans la faible gamme des largeurs de cernes. En effet, $94 \%$ des largeurs d'accroissement à $1,30 \mathrm{~m}$ étaient com- prises entre 0,5 et $3 \mathrm{~mm}$. La moyenne des accroissements annuels pour les 24 arbres, à $1,30 \mathrm{~m}$, est de $1,74 \mathrm{~mm}$. Cette remarque renseigne sur le domaine de validité de nos modèles.

Un descriptif dendrométrique simple des 24 arbres de l'étude est donné dans le tableau I.

Tableau I. Données dendrométriques simples des 24 chênes rouvres utilisés dans l'étude.

\begin{tabular}{|c|c|c|c|c|c|c|}
\hline \multirow{2}{*}{ Forêt } & \multirow{2}{*}{$\begin{array}{c}\text { Arbre } \\
\text { (code labo) }\end{array}$} & \multirow{2}{*}{$\begin{array}{c}\text { Diam } \\
(e n \mathrm{~cm})\end{array}$} & \multirow{2}{*}{$\begin{array}{l}\text { Haut } \\
(e n m)\end{array}$} & \multicolumn{2}{|c|}{ Nombre de cernes } & \multirow{2}{*}{$\begin{array}{c}\text { Larg } \\
(e n \mathrm{~mm})\end{array}$} \\
\hline & & & & à $4,40 \mathrm{~m}$ & à $1,30 \mathrm{~m}$ & \\
\hline \multicolumn{7}{|l|}{ Lorraine } \\
\hline \multirow[t]{2}{*}{ Mondon } & 2368 & 48 & 27,0 & 150 & 144 & 1,53 \\
\hline & 2369 & 58 & 28,9 & 151 & 150 & 1,84 \\
\hline \multirow[t]{2}{*}{ Bezange } & 2370 & 85 & 24,0 & 207 & 206 & 1,91 \\
\hline & 2371 & 102 & 26,1 & 208 & 207 & 2,26 \\
\hline \multirow[t]{2}{*}{ Bride } & 2372 & 61 & 23,0 & creux * & 209 & 1,38 \\
\hline & 2373 & 55 & 30,5 & creux * & 201 & 1,28 \\
\hline \multicolumn{7}{|l|}{ Alsace } \\
\hline \multirow[t]{2}{*}{ Saverne } & 2374 & 79 & 32,3 & 176 & 170 & 2,12 \\
\hline & 2375 & 65 & 33,1 & 166 & 159 & 1,88 \\
\hline \multirow[t]{2}{*}{ Bouxwiller } & 2376 & 67 & 35,9 & 190 & 188 & 1,62 \\
\hline & 2377 & 71 & 35,5 & 186 & 183 & 1,78 \\
\hline \multirow[t]{2}{*}{ Oberbronn } & 2378 & 52 & 34,2 & 134 & 131 & 1,87 \\
\hline & 2379 & 50 & 33,8 & 126 & 118 & 1,99 \\
\hline \multirow{2}{*}{ Reischoffen } & 2380 & 49 & 35,2 & 181 & 177 & 1,31 \\
\hline & 2381 & 53 & 35,8 & 186 & 181 & 1,38 \\
\hline \multirow[t]{2}{*}{ Nonnenhart } & 2382 & 59 & 34,8 & 153 & 151 & 1,79 \\
\hline & 2383 & 58 & 35,1 & 154 & 152 & 1,76 \\
\hline \multirow[t]{2}{*}{ Steinbach } & 2384 & 71 & 29,7 & 184 & 181 & 1,82 \\
\hline & 2385 & 70 & 31,8 & 181 & 181 & 1,81 \\
\hline \multirow[t]{6}{*}{ Haguenau } & 2386 & 53 & 33,0 & 158 & 155 & 1,59 \\
\hline & 2387 & 47 & 31,2 & 159 & 157 & 1,38 \\
\hline & 2388 & 56 & 32,6 & 145 & 143 & 1,85 \\
\hline & 2389 & 49 & 32,0 & 145 & 142 & 1,63 \\
\hline & 2390 & 82 & 35,4 & 173 & 171 & 2,17 \\
\hline & 2391 & 70 & 35,5 & 174 & 170 & 1,90 \\
\hline
\end{tabular}

Diam : diamètre sur écorce à 1,30 m; Haut : hauteur totale de l'arbre ; Larg : largeur moyenne des cernes à 1,30 m ; *le nombre de cernes à $0,40 \mathrm{~m}$ pour ces deux arbres a été estimé à partir de leur nombre de cernes à $1,30 \mathrm{~m}$ et du nombre d'années nécessaires pour la moyenne des 22 autres arbres pour croître de 0,40 à $1,30 \mathrm{~m}$ de hauteur. 
À l'intérieur de chaque grume, nous disposions a priori de plusieurs niveaux, les mêmes pour chaque arbre. Les niveaux échantillonnés se trouvaient toujours sous le premier gros défaut de la bille de pied. Les niveaux retenus pour l'étude étaient les suivants : No $(0,40 \mathrm{~m}), \mathrm{N} 1$ $(1,30 \mathrm{~m}), \mathrm{N} 2(3,50 \mathrm{~m}), \mathrm{N} 3(6,30 \mathrm{~m}), \mathrm{N} 4(9,10 \mathrm{~m})$, N5 $(11,90 \mathrm{~m}), \mathrm{N} 6(14,70 \mathrm{~m})$

Compte tenu de la lourdeur de cet échantillonnage, nous avons diminué le nombre de niveaux à considérer par arbre au fur et à mesure de l'avancement de notre travail.

Pour un niveau donné, nous disposions d'une éprouvette du côté le plus long et de son opposé ; pour réduire le matériel à traiter et pour éviter d'éventuels problèmes de bois de tension, nous avons choisi de travailler sur le côté « opposé », court.

Les profils microdensitométriques sur lesquels s'appuient nos modèles ont été recueillis sur des barrettes épaisses de $2 \mathrm{~mm}$, présentant le plan radial-tangentiel et dont l'humidité était stabilisée à $11,8 \%$. Un programme d'acquisition automatique des données microdensitométriques nous délivrait, pour chaque cerne, sa largeur (LC), les largeurs des zones de bois initial et de bois final (LBI et LBF) et les densités de ces deux zones (DBI, DBF). Pour un niveau donné, un cerne était repéré par son âge compté depuis la moelle (âge cambial). La limite entre le bois initial et le bois final pour le calcul des densités des deux zones est posée pour le point de densité égale à :

$$
\mathrm{D}_{\text {limite }}=\frac{\mathrm{D}_{\min }+\mathrm{D}_{\max }}{2}
$$

où $\mathrm{D}_{\min }$ et $\mathrm{D}_{\max }$ sont respectivement les densités minimales et maximales du cerne.

Chaque valeur de densité délivrée par le microdensitomètre était corrigée de telle manière que la densité moyenne de chaque barrette obtenue par microdensitométrie soit strictement égale à la masse volumique de la barrette mesurée, au même taux d'humidité, par le rapport entre sa masse et son volume.

\section{RÉSULTATS ET DISCUSSION}

\section{Étude de la variabilité intra-arbre}

\section{Modèle de densité moyenne de cerne et importance relative des variables explicatives}

Nous devions étudier la variabilité de la densité dans l'arbre en nous appuyant sur le profil microdensitométrique de chaque cerne repéré par sa largeur, son âge cambial et sa hauteur. II nous fallait donc choisir un modèle décrivant au mieux ce profil sans que le traitement informatique et statistique des données ne soit trop lourd. Compte tenu du fait que le bois de chêne est un bois à zone initiale poreuse et que la limite entre le bois initial et final est relativement bien tranchée, nous avons réduit le profil en une fonction à deux paliers définie par la densité du bois initial (DBI), par la densité du bois final (DBF) et par les largeurs respectives de ces deux zones (LBI et LBF). D'où un modèle de densité moyenne du cerne $\left(D_{\text {moy }}\right)$ :

$$
\mathrm{D}_{\text {moy }}=\frac{\mathrm{LBI}}{\mathrm{LC}} \times \mathrm{DBI}+\frac{\mathrm{LBF}}{\mathrm{LC}} \times \mathrm{DBF}
$$

C'est sur les composantes DBI, DBF, LBI et LBF du modèle [1] que nous avons étudié la variabilité intra- et interarbre.

Afin de cerner l'importance relative des variables explicatives à notre disposition (âge cambial [âge], largeur de cerne [LC] et niveau [N]) sur la variabilité des composantes microdensitométriques, nous avons tout d'abord réalisé une analyse en composantes principales sur les arbres 2376 et 2372 , dont nous avions recueilli les composantes microdensitométriques à tous les niveaux (sept niveaux pour l'arbre 2376, six pour l'arbre 2372). Nous avons recherché la contribution de nos trois variables explicatives sur les axes décrivant la variabilité des composantes de densité.

Nous avons réalisé une ACP pour chacun des deux arbres. On retrouve les fortes corrélations d'âge et de LC sur les deux premiers axes de variabilité, dont ils expliquent plus de $70 \%$. Pour l'âge cambial, la corrélation est de 0,76 pour l'arbre 2376 sur l'axe 1 et de 0,66 pour l'arbre 2372 sur l'axe 2 . Pour la largeur de cerne, la corrélation est de 0,72 pour l'arbre 2376 sur l'axe 1 et de 0,64 sur l'axe 2 ; dans le cas de l'arbre 2372 , les corrélations de LC sur les axes 1 et 2 sont respectivement de 0,68 et 0,70 . 
Le niveau n'est fortement corrélé qu'avec l'axe 3 de l'ACP $(0,82$ pour l'arbre 2376 et 0,95 pour l'arbre 2372 ) ; cet axe représente $10 \%$ de la variabilité.

II semble donc que l'âge cambial et que la largeur de cerne soient les variables explicatives principales de la variabilité des composantes microdensitométriques dans l'arbre (DBI, DBF, LBI et LBF), le niveau ayant une importance relativement secondaire. Nous avons donc établi nos modèles en fonction de l'âge compté depuis la moelle (Âge) et de la largeur de cerne (LC), en ne nous intéressant qu'au niveau $1,30 \mathrm{~m}$ dans un premier temps.

\section{Modèles de largeur de bois initial et de bois final à $1,30 \mathrm{~m}$}

La figure 1 illustre pour l'arbre 2376, représentatif des 24 chênes de l'étude, la relation linéaire entre la largeur du bois final et la largeur de cerne ; on remarque aussi la quasi-constance de la largeur du bois initial quelle que soit la largeur de cerne. Étant donné la richesse en tissus fibreux, denses du bois final, cette relation induit une augmentation automatique de la densité du cerne quand la largeur d'accroissement augmente à l'intérieur de l'arbre. Rappe- lons que nous travaillons sur une gamme de faibles largeurs de cernes. On retrouve en cela les résultats des travaux de Polge et Keller (1973).

Nous écrivons donc :

$$
\mathrm{LB} \mid=\text { Constante }=a
$$

Comme :

$$
L B I+L B F=L C
$$

on a :

$$
\mathrm{LBF}=\mathrm{LC}-\mathrm{a}
$$

Les paramètres des modèles obtenus " arbre " par " arbre ", pour la hauteur $1,30 \mathrm{~m}$, sont indiqués dans le tableau II. En plus des paramètres " $a$ ", on y trouvera également les paramètres des modèles de densité du bois initial (DBI) et du bois final (DBF), les écarts types résiduels (sr) et les coefficients de détermination $\left(R^{2}\right)$ attachés à chacun de ces modèles.

Pour l'ensemble des arbres, le modèle de largeur de bois final en fonction de la largeur de cerne présente de très bon coefficients de détermination (le $R^{2}$ moyen pour les 24 arbres considérés à $1,30 \mathrm{~m}$ est de $0,94)$, malgré des écarts types résiduels relativement importants (la moyenne des écarts types résiduels pour les 24 arbres

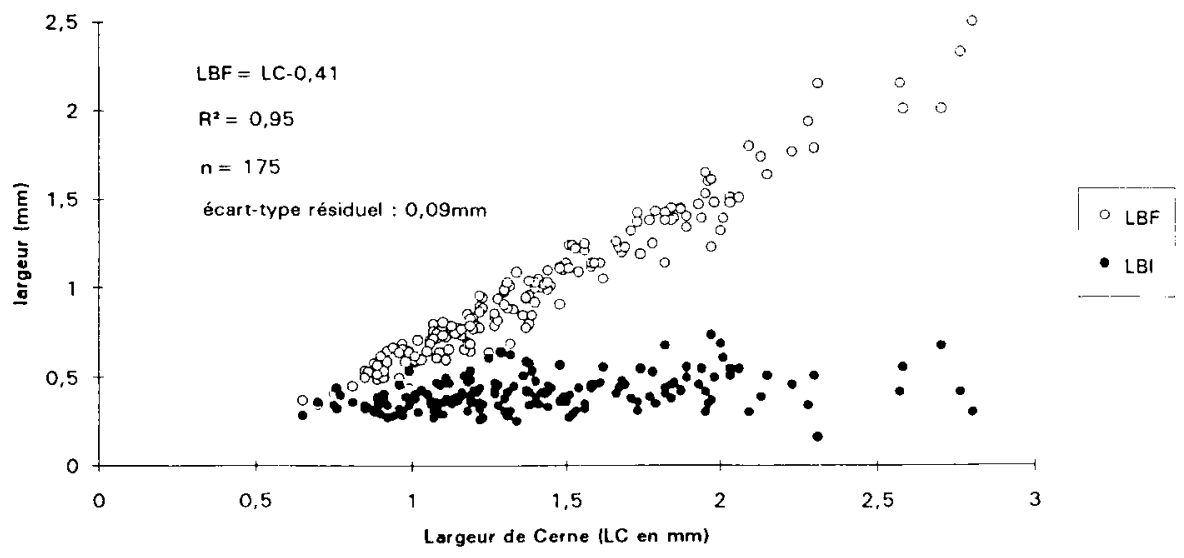

Fig 1. Évolution des largeurs du bois initial (LBI) et du bois final (LBF) en fonction de la largeur de cerne pour l'arbre 2376 à $1,30 \mathrm{~m}$. 
Tableau II. Valeurs des paramètres, écarts types résiduels (sr) et $R^{2}$ des modèles de largeur de bois final, de densité du bois initial et de densité du bois final obtenus à $1,30 \mathrm{~m}$ pour les 24 chênes rouvres utilisés dans l'étude.

\begin{tabular}{rcccccccccccc}
\hline Arbre & $\mathrm{n}$ & $\mathrm{a}$ & $\mathrm{srLBF}$ & $\mathrm{R}^{2}$ & $\mathrm{~g}$ & $\mathrm{~d}$ & $\mathrm{srDBI}$ & $\mathrm{R}^{2}$ & $\gamma$ & \multicolumn{1}{c}{$\delta$} & $s r D B F$ & $\mathrm{R}^{2}$ \\
\hline 2368 & 111 & 0,45 & 0,12 & 0,97 & -15 & 564 & 44 & 0,26 & -16 & 839 & 58 & 0,20 \\
2369 & 143 & 0,39 & 0,10 & 0,88 & -14 & 557 & 65 & 0,24 & -11 & 836 & 82 & 0,10 \\
2370 & 200 & 0,46 & 0,11 & 0,96 & -13 & 652 & 62 & 0,32 & -21 & 1013 & 83 & 0,40 \\
2371 & 203 & 0,58 & 0,24 & 0,91 & -5 & 522 & 63 & 0,06 & -20 & 892 & 68 & 0,11 \\
2372 & 208 & 0,36 & 0,10 & 0,90 & -19 & 574 & 57 & 0,55 & -12 & 803 & 61 & 0,32 \\
2373 & 198 & 0,31 & 0,11 & 0,93 & -9 & 640 & 77 & 0,13 & -7 & 888 & 66 & 0,10 \\
2374 & 166 & 0,49 & 0,19 & 0,91 & -14 & 615 & 63 & 0,28 & -25 & 1001 & 65 & 0,52 \\
2375 & 146 & 0,38 & 0,10 & 0,95 & -26 & 743 & 65 & 0,51 & -28 & 979 & 59 & 0,76 \\
2376 & 175 & 0,41 & 0,09 & 0,95 & -20 & 648 & 70 & 0,39 & -27 & 990 & 70 & 0,52 \\
2377 & 174 & 0,51 & 0,12 & 0,93 & -4 & 454 & 62 & 0,03 & -17 & 900 & 60 & 0,41 \\
2378 & 127 & 0,42 & 0,10 & 0,94 & -19 & 631 & 50 & 0,48 & -36 & 1131 & 67 & 0,65 \\
2379 & 109 & 0,42 & 0,12 & 0,97 & -7 & 545 & 58 & 0,06 & -35 & 1029 & 60 & 0,60 \\
2380 & 175 & 0,36 & 0,10 & 0,88 & -28 & 742 & 41 & 0,80 & -27 & 1017 & 52 & 0,63 \\
2381 & 174 & 0,39 & 0,10 & 0,94 & -34 & 883 & 72 & 0,65 & -32 & 1130 & 73 & 0,62 \\
2382 & 148 & 0,40 & 0,13 & 0,95 & -10 & 594 & 60 & 0,18 & -8 & 926 & 59 & 0,12 \\
2383 & 117 & 0,42 & 0,11 & 0,93 & -36 & 775 & 75 & 0,56 & -31 & 1027 & 74 & 0,50 \\
2384 & 168 & 0,38 & 0,12 & 0,97 & -19 & 722 & 67 & 0,36 & -22 & 1015 & 52 & 0,57 \\
2385 & 172 & 0,41 & 0,12 & 0,96 & -11 & 558 & 57 & 0,25 & -16 & 844 & 49 & 0,46 \\
2386 & 144 & 0,43 & 0,11 & 0,91 & -24 & 709 & 59 & 0,50 & -27 & 1040 & 54 & 0,61 \\
2387 & 151 & 0,38 & 0,08 & 0,97 & -18 & 710 & 68 & 0,35 & -28 & 1087 & 67 & 0,56 \\
2388 & 133 & 0,45 & 0,11 & 0,95 & -17 & 673 & 67 & 0,26 & -19 & 1001 & 44 & 0,53 \\
2389 & 131 & 0,41 & 0,09 & 0,94 & -12 & 596 & 63 & 0,17 & -11 & 915 & 57 & 0,16 \\
2390 & 133 & 0,48 & 0,14 & 0,94 & -18 & 660 & 86 & 0,15 & -32 & 1006 & 68 & 0,45 \\
2391 & 132 & 0,40 & 0,10 & 0,91 & -8 & 535 & 68 & 0,10 & -20 & 961 & 63 & 0,43 \\
Moyennne & 0,42 & 0,12 & 0,94 & -17 & 638 & 63 & 0,32 & -22 & 970 & 63 & 0,43 \\
& & & & & & & & & & & &
\end{tabular}

On rappelle que $: \mathrm{LBF}=\mathrm{LC}-a ; \mathrm{BDI}=g \times \sqrt{A g e+d} ; \mathrm{DBF}=g \times \sqrt{A g e+\delta}$. Avec LC, Age, LBF, DBI et DBF respectivement largeur de cerne, âge cambial, largeur de bois final, densité du bois initial et densité du bois final ; $n$ : nombre d'observations; $a$ et $s r L B F$ sont exprimés en $\mathrm{mm} ; d$ et $\delta$, $s r \mathrm{DBI}$ et $s r \mathrm{DBF}$ sont exprimés en $\mathrm{kg} / \mathrm{m}^{3} ; g$ et $\gamma$ sont exprimés en $\mathrm{kg} / \mathrm{m}^{3} / a n n e ́ e^{1 / 2}$; les extrêmes observés pour chacun des coefficients sont écrits en caractères gras.

considérés à $1,30 \mathrm{~m}$ est de $0,12 \mathrm{~mm}$ ). Les paramètres « $a$ » obtenus à 1,30 m pour chaque arbre varient sur une population de 24 chênes, de 0,31 à $0,58 \mathrm{~mm}$, ce qui est relativement important. Ce paramètre peut être interprété comme une largeur de bois initial. Son écart type est de 0,01 pour quasiment tous les chênes étudiés à hauteur d'homme. La précision relative de ce paramètre, sur lequel s'appuiera notre étude de l'effet " niveau " et des effets individuels, est correcte. En effet, les coefficients de variation (rapport de l'écart type sur la valeur estimée) de ce paramètre pour les 24 arbres à hauteur d'homme varient de 2 à $5 \%$. Il faut bien noter qu'une bonne précision statistique du paramètre " a ", comme celle des paramètres des modèles de densité du bois initial et du bois final, est rendue nécessaire par son utilisation ultérieure dans l'analyse des effets hauteurs et des effets individuels. 


\section{Modèles de densité du bois initial et du bois final à $1,30 \mathrm{~m}$}

Pour l'établissement de ces modèles de densité du bois initial (DBI) et de densité du bois final (DBF), nous avons appliqué le formalisme suivant :

$$
\begin{aligned}
& \mathrm{DBI}=g \times \sqrt{\mathrm{A} g \mathrm{e}+d} \\
& \mathrm{DBF}=\gamma \times \sqrt{\hat{A} g \mathrm{e}+\delta}
\end{aligned}
$$

où $g$ et $\gamma$ sont de signe négatif, $d$ et $\delta$ sont positifs.

Ce formalisme rend bien compte de l'évolution rapide des propriétés du bois vers la moelle, comme l'ont montré les travaux d'Ohtani et Fukazawa (1972) et de Fukazawa (1984). Les travaux d'Huber (1991) et d'Eyono Owoundi (1992) avaient mis en évidence une évolution linéaire de la densité tout au long de sa croissance radiale; le modèle que nous proposons rend également compte de ce phénomène bien que ces variations soient plus faibles vers la périphérie du tronc. La figure 2 illustre l'application du modèle de densité de bois initial au cas de l'arbre 2380. On notera que l'effet de l'âge se maintient au-delà de 100 ans.

Nous nous sommes assurés par une étude des résidus des modèles [5] et [6] que l'introduction d'un effet « largeur de cerne » n'améliorait pas la précision des modèles.

La réponse des arbres aux modèles de $\mathrm{DBI}$ est très variable. Pour trois arbres de notre étude (les arbre 2371, 2377 et 2379), il n'y a quasiment pas de relation statistiquement significative entre $\mathrm{DBI}$ et l'âge. Pour la plupart de nos arbres, la réponse à l'effet de l'âge est caractérisée par un $R^{2}$ moyen de l'ordre de 0,30 à 0,50 . Pour quelques arbres (les arbres 2380 et 2381 ), la réponse est très nette et l'effet de l'âge se fait encore bien sentir près de l'écorce. II faut noter d'une façon générale l'importance et la variabilité des écarts types autour des modèles ajustés par arbre ; ceuxci sont compris entre 41 et $86 \mathrm{~kg} / \mathrm{m}^{3}$. Pour expliquer cette importante variabilité résiduelle, on peut invoquer les variations interannuelles d'ordre climatique mais aussi et surtout les problèmes de mesure de la densité du bois de chêne liés à sa grande complexité anatomique, que nous ne déve-

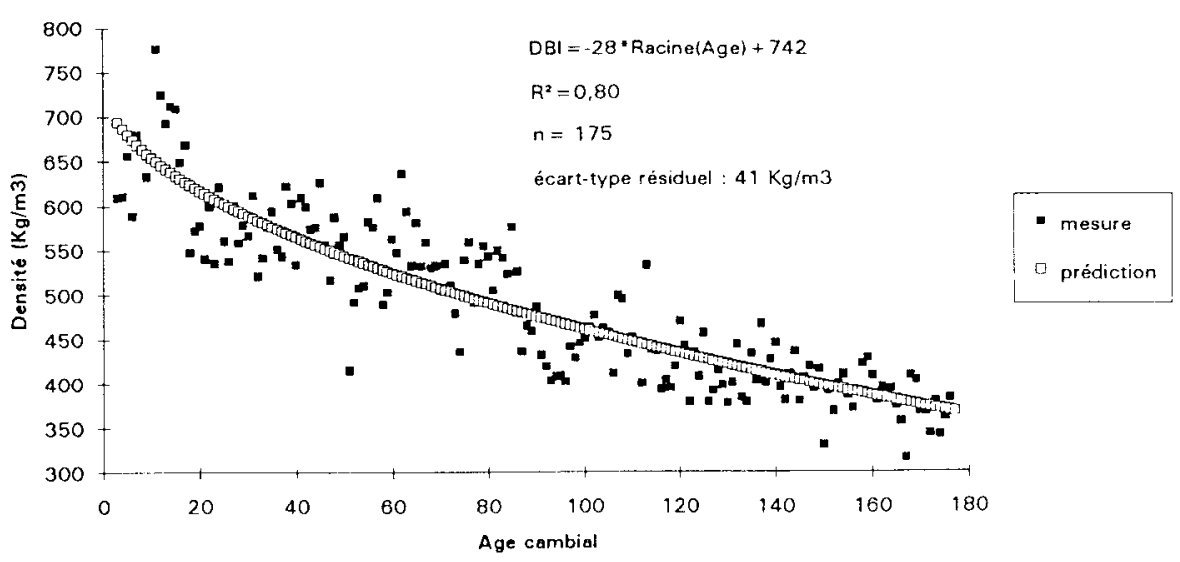

Fig 2. Évolution de la densité de bois initial (DBI) en fonction de l'âge cambial pour l'arbre 2380 à $1,30 \mathrm{~m}$. 
lopperons pas dans le présent article. En ce qui concerne les paramètres, on peut noter une importante variabilité du paramètre « $g$ » selon les arbres, qui est à relier à la variabilité déjà évoquée des $R^{2}, g$ varie entre -4 et -36 , avec une moyenne de -17 . La précision absolue de ce paramètre est pour tous les arbres de l'ordre de 2,0; compte tenu de sa variabilité, la précision relative rendue par le coefficient de variation est très variable (de 4 à $43 \%$ avec une moyenne de $15 \%$ ). Pour tous les arbres, le paramètre " $d$ " est statistiquement assez fiable. Pour notre échantillonnage, ce paramètre a des valeurs comprises entre 522 et 883 avec une moyenne de 638 . Son écart type moyen pour 24 arbres à $1,30 \mathrm{~m}$ est de 19.

Pour les modèles de DBF, on peut reprendre les mêmes remarques que pour DBI. Là aussi, les $R^{2}$ varient de façon importante (de 0,10 à 0,76 ). Les écarts types résiduels sont forts, ils vont de 41 à $83 \mathrm{~kg} / \mathrm{m}^{3}$. Le paramètre " $\gamma$ ", homologue à " $g$ ", varie de -7 à -36 avec une moyenne de -22 ; il présente, en moyenne, un écart type de 1,9 et le coefficient de variation moyen de ce paramètre est de $11 \%$ (sur 24 arbres à $1,30 \mathrm{~m}$, il varie de 6 à $23 \%$ ). Pour " $\delta$ », les valeurs vont de 836 à 1131 avec une moyenne de 970 . Comme pour " $d$ ", l'écart type des paramètres « $\delta$ » ajustés arbre par arbre est de 19, ces paramètres sont donc assez précisément estimés pour l'ensemble des arbres.

\section{Reconstitution du modèle de densité moyenne de cerne à $1,30 \mathrm{~m}$}

À partir des modèles de largeur du bois final, de densité du bois final et de densité du bois initial et en rappelant le modèle [1], il est possible d'écrire un modèle de densité moyenne du cerne $\left(D_{\text {may }}\right)$ :

$$
D_{\text {mioy }}=1+J \times \frac{1}{L C}+K \times \sqrt{A g e}+L \times \sqrt{\frac{A g e}{L C}}
$$

Les paramètres I, J, $K$ et $L$ dépendent de l'arbre. Ces paramètres se déduisent des paramètres des modèles [4], [5] et [6], d'après les formules suivantes:

$$
\begin{aligned}
& \mathrm{I}=\delta \\
& \mathrm{J}=a \times(d-\delta) \\
& \mathrm{K}=\gamma \\
& \mathrm{L}=a \times(g-\gamma)
\end{aligned}
$$

Le modèle reconstitué, qui explique statistiquement la densité moyenne du cerne par la largeur de cerne et par l'âge cambial, présente une colinéarité importante lors de son ajustement en bloc par régression linéaire multiple. Cette colinéarité dégrade la qualité des paramètres $\mathrm{I}, \mathrm{J}, \mathrm{K}$ et $\mathrm{L}$ de l'équation lorsqu'ils sont ajustés par ce biais ; leur interprétation devient donc quasiment impossible (Degron, 1994). II nous semble donc préférable pour étudier la variabilité des arbres, exprimées par leurs modèles, de travailler sur les modèles [4], [5] et [6], qui sont de meilleure qualité statistique.

Nous retiendrons de ces résultats, résumés dans le tableau II, qu'à largeur de cerne et âge depuis la moelle fixés la variabilité entre arbres pour la largeur du bois initial, la densité du bois initial, la densité du bois final et par voie de conséquence, la densité moyenne de cerne est importante. Chez le chêne rouvre, un même scénario sylvicole appliqué à des individus différents conduira à la production de bois de densités sensiblement différentes tant sur le plan de leur moyenne que de leur distribution. Ces résultats sont conformes à ceux obtenus par Polge et Keller (1973) sur $Q$ petraea et $Q$ robur, Zhang et al (1993) sur $Q$ robur et $Q$ petrae et Ackermann (1995) sur $Q$ robur.

\section{Effet du niveau dans l'arbre sur les paramètres des modèles}

Sur dix arbres pour lesquels nous disposions de mesures microdensitométriques (DBI, DBF, LBI et LBF) à trois niveaux $(0,40 \mathrm{~m}-1,30 \mathrm{~m}-3,50 \mathrm{~m})$, nous avons réalisé une analyse de la variance sur les paramètres des différents modèles présentés 
Tableau III. Analyse de la variance sur les effets " niveau " et " arbre " sur les paramètres des modèles de densité du bois initial (DBI), densité du bois final (DBF) et largeur de bois final (LBF) établis pour 10 chênes rouvres de l'étude à trois hauteurs différentes $(0,40,1,30$ et $3,50 \mathrm{~m})$

\begin{tabular}{|c|c|c|c|c|c|c|}
\hline Effet & $D D L$ & a & g & d & $\gamma$ & $\delta$ \\
\hline Arbre & 9 & $4,42^{\star \star}$ & 2,53 * & $3,15^{*}$ & $5,69 * * \star$ & $6,27^{\star \star \star}$ \\
\hline Niveau & 2 & $5,24^{*}$ & $4,16^{*}$ & $15,30^{* \star \star}$ & $6,32^{\star \star}$ & $10,2^{\star \star}$ \\
\hline Erreur & 18 & & & & & \\
\hline
\end{tabular}

Niveau de signification du test de Fisher : ns : non significatif ; *significatif à $5 \%$, "*significatif à $1 \%$, ${ }^{\star \star \star *}$ significatif à $0,1 \%$.

plus haut et qui avaient été établis pour chaque arbre et pour chaque niveau. Le tableau III donne les résultats de l'analyse de la variance et le seuil de signification de l'effet " niveau " sur les paramètres.

On remarque un effet du niveau, plus ou moins fort suivant les paramètres considérés, mais toujours significatif. Une comparaison des moyennes (test de Duncan) par niveau pour les paramètres des modèles de LBF, DBI et DBF est présentée dans le tableau IV. Cette comparaison permet de montrer la place particulière du niveau $0,40 \mathrm{~m}$ : les paramètres pour cette hauteur ont statistiquement des valeurs absolues plus fortes qu'aux autres hauteurs.

On aura également remarqué un effet " arbre " significatif sur les paramètres des trois modèles auxquels nous nous sommes intéressés. Ceci n'est pas surprenant si l'on se reporte aux données du tableau II. Cet effet « arbre " est particulièrement important sur la largeur du bois initial a $\left(F(9,18)=4,42^{\star \star}\right)$ et les deux paramètres du modèle de densité du bois final
( $\gamma$ et $\delta$ avec des $F(9,18)$ respectivement de $5,69^{\star \star \star}$ et $\left.6,27^{\star \star \star}\right)$. En revanche, l'effet « arbre " n'est significatif qu'à $5 \%$ sur les paramètres du modèle de densité du bois initial $\left(F(9,18)=2,53^{\star}\right.$ et $3,15^{\star}$ respectivement pour $g$ et $d$ ).

\section{Étude de la variabilité interarbre}

Sur 20 chênes dont les modèles ont pu être établis à 0,40 et $1,30 \mathrm{~m}$ (ie, ceux du tableau I, à l'exception des arbres 2380, 2381, 2384 et 2385 pour lesquels les barettes à $0,40 \mathrm{~m}$ ont éclaté), nous avons analysé la variabilité des paramètres des modèles [4], [5] et [6]. Notons que cette méthode, qui consiste à étudier les paramètres des équations de régression, est relativement originale dans le domaine d'étude de la qualité des bois et qu'elle permet d'évaluer des différences de forme de modèles entre les individus. La variabilité a été décomposée en un effet du site, de l'arbre dans le site, d'une interaction entre le site et le niveau et d'un effet « niveau " compte tenu de l'importance

Tableau IV. Comparaison des moyennes par niveau pour les paramètres des modèles de largeur de bois final $(a)$, densité du bois initial ( $g$ et $d$ ) et densité du bois final $(\gamma$ et $\delta$ )

\begin{tabular}{lccccc}
\hline Niveau $(\mathrm{e} n \mathrm{~m})$ & $\mathrm{a}$ & $\mathrm{g}$ & $\mathrm{d}$ & $\gamma$ & $\delta$ \\
\hline 0,40 & 0,44 & -20 & 696 & -24 & $\mathbf{9 8 3}$ \\
1,30 & 0,40 & -12 & 576 & -18 & 922 \\
3,50 & 0,38 & -10 & 529 & -14 & 866 \\
\hline
\end{tabular}

Les chiffre en gras indiquent que le niveau $0,40 \mathrm{~m}$ se détache des autres statistiquement par utilisation du test de Duncan. 
Tableau V. Analyse de la variance sur les effets " site ", " arbre dans site ", " niveau " et interaction " site-niveau " sur les paramètres des modèles de largeur de bois final $(a)$, densité du bois initial ( $g$ et $\alpha$ ) et densité de bois final ( $\gamma$ et $\delta$ ) établis pour 20 chênes rouvres de l'étude à deux niveaux différents $(0,40$ et $1,30 \mathrm{~m})$ et représentés sur dix sites (deux arbres par site).

\begin{tabular}{lrlrrrr}
\hline Effet & $D D L$ & \multicolumn{1}{c}{$\mathrm{a}$} & $\mathrm{g}$ & $\mathrm{d}$ & $\gamma$ & $\delta$ \\
\hline Site & 9 & $3,48^{*}$ & $2,22 \mathrm{~ns}$ & $1,29 \mathrm{~ns}$ & $5,10^{* *}$ & $1,82 \mathrm{~ns}$ \\
Arbre (site) & 10 & $2,04 \mathrm{~ns}$ & $1,11 \mathrm{~ns}$ & $1,91 \mathrm{~ns}$ & $1,00 \mathrm{~ns}$ & $3,21^{* *}$ \\
Niveau & 1 & $4,64^{*}$ & $4,89^{*}$ & $15,17^{\star \star}$ & $6,86^{*}$ & $8,81^{\star \star}$ \\
Site-niveau & 9 & $3,16^{*}$ & $1,39 \mathrm{~ns}$ & $1,31 \mathrm{~ns}$ & $1,14 \mathrm{~ns}$ & $1,65 \mathrm{~ns}$ \\
Erreur & 10 & & & & &
\end{tabular}

ns : non significatif ; " significatif à $5 \%$; ${ }^{* \star}$ significatif à $1 \%$. ${ }^{* \star}$ significatif à $0,1 \%$.

particulière du niveau le plus bas. Le tableau $V$ présente les résultats de l'analyse de variance sur ces effets.

On retrouve comme pour le tableau III, un effet « arbre dans site " relativement important $\left(F(10,10)=3,21^{* *}\right)$ sur la constante « $\delta$ du modèle de densité du bois final. On note également un effet significatif du site sur la largeur du bois initial ( $F$ $(9,10)=3,48^{*}$ ) et sur le paramètres « $\gamma$ " du modèle de densité du bois final en fonction de l'âge.

En revanche, aucun effet "site », ni aucun effet « arbre dans site » n'apparaît pour les paramètres « $g$ » et « $d$ " du modèle donnant la densité du bois initial. Ceci est relativement cohérent avec les commentaires que nous émettions au sujet du tableau III à propos de l'effet " arbre » sur les paramètres " $g$ " et « $d$ »: dans ce tableau, pour dix arbres seulement et tous sites confondus, l'effet " arbre " sur " $g$ " et « $d$ » n'était significatif qu'au seuil de $5 \%$.

L'effet « niveau » entre 0,40 et 1,30 m est confirmé (cf tableau III). L'interaction entre le site et le niveau semble très faible; elle n'est significative qu'au seuil de $5 \%$ pour le paramètre « $a$ » $\left(F(9,10)=3,16^{\star}\right)$.

Les résultats de notre analyse portant sur les effets « site " et « arbre dans site » doivent être relativisés compte tenu du faible nombre d'arbres étudiés et du manque de données caractérisant leur station d'origine. II faut donc rester circonspect quant à l'interprétation de ces résultats et retenir pour le moins un effet interindividuel important sur les paramètres des modèles de densité du bois de chêne rouvre; ce résultat est relativement conforme aux conclusions d'Ackermann (1995) sur la variabilité interindividuelle de la densité du bois chez le chêne pédonculé.

À raison de trois stations forestières et sur la base d'un échantillonnage de 35 arbres par station, cet auteur avait par ailleurs mis en évidence un effet de la station sur les composantes microdensitométriques à largeur de cerne et âge fixés. L'effet « station " était significatif sur la largeur de bois initial, sur la densité du bois initial et sur la densité du bois final. La densité du bois final était plus sensible à l'effet « station », que la densité du bois initial.

\section{CONCLUSION ET PERSPECTIVES}

Sur un échantillonnage de 24 chênes rouvres représentés par plusieurs niveaux par arbre, nous avons étudié la variabilité intraindividuelle et secondairement la variabilité interindividuelle des composantes de la densité moyenne du cerne : largeur du bois final, densité du bois final et densité du bois initial. 
Nous avons trouvé que, dans l'arbre, la largeur du bois initial pouvait être considérée constante et que la densité du bois initial et du bois final ne dépendaient que de l'âge. Nous avons montré un effet du niveau dans l'arbre, à âge et largeur de cerne fixés, concentré sur le niveau le plus bas de notre échantillonnage. Pour ce faire nous avons étudié la variabilité des paramètres des modèles de densité.

En travaillant sur ces mêmes paramètres, nous avons analysé la variabilité interindividuelle. Nous avons montré des effets " site " et « arbre dans site ". Compte tenu de la faiblesse de notre échantillonnage, il est difficile d'interpréter ces effets, ils confirment tout au plus l'importance de la varia- bilité entre les arbres de la densité du bois, à largeur de cerne et âge fixés.

La méthode basée sur l'étude de la variabilité des paramètres que nous avons développés semble en tout cas riche en informations. Après le travail d'Ackermann (1995), qui a mis au jour certaines relations entre le milieu et les composantes de la densité du bois, à âge et largeur de cerne fixés, il serait intéressant de confirmer et de préciser ces relations en étudiant l'effet de la station sur les paramètres décrivant la variabilité intra-arbre de la densité du bois. L'utilisation de cette méthode sur un échantillonnage dépassant largement celui de notre étude serait sans doute riche d'enseignement dans le cadre d'une étude " station-qualité du bois ".

Par ailleurs, il serait sans doute intéressant de tenter d'expliquer la variabilité résiduelle demeurant autour des modèles liés à chaque arbre mis à jour : nous pensons qu'il faudrait notamment se pencher sur un possible effet $\mathrm{du}$ « bois de tension » qui pourrait induire une variabilité circonférencielle de densité du bois à cerne fixé mais également sur les effets climatiques annuels susceptibles à largeur de cerne fixée d'engendrer des différences de structure anatomique et partant de densité du bois.

\section{REMERCIEMENTS}

Ce travail a été supporté financièrement par I'ONF dans le cadre d'une convention quinquennale avec l'Inra portant sur la sylviculture et la qualité du bois de chêne. Cette étude a également été soutenue par l'UE, elle s'intègre dans le projet « Forest Planning and Management Tools " PL 920715. Nous remercions tout spécialement $P$ Gelhaye pour la préparation du matériel de notre étude.

\section{RÉFÉRENCES}

Ackermann F (1995) Étude de l'influence du type de station forestière sur la qualité du bois de chêne pédonculé (Quercus robur) dans les chênaies de l'Adour et des coteaux basco-béarnais. Ann Sci For 52, 635652

Degron $R$ (1994) Modélisation intracerne de la densité du bois de chêne rouvre (Quercus petraea Liebl) en fonction de la largeur de cerne et de la position dans l'arbre. Application à l'évaluation des effets sylvicoles sur la densité du bois et à une sélection réaliste des arbres de qualité. DEA, Inra, Nancy, 42 p

De Hercé E (1991) Ėbauche d'un modèle de croissance du chêne rouvre à partir de données de placettes permanentes. DEA, Engref, $21 p$

Eyono Owoundi $R$ (1991) Modélisation de la rétractibilité du bois en relation avec des paramètres de la structure de l'accroissement annuel et de la position dans l'arbre chez Quercus robur L et $Q$ petraea Liebl. Application à l'intégration de la rétractibilité du bois dans les modèles de croissance de peuplements et d'arbres. Thèse, Inra-Engref, $196 p+$ annexes

Ferrand JC (1982) Réflexions sur la densité du bois I. Définition de la densité du bois. Holzforschung 2, 99-105

Fukazawa $\mathrm{K}$, Ohtani $\mathbf{J}$ (1972) The effects of age in density variation within one stem of Quercus crispula. Research Bulletins of the College Experiment Forests, Hokkaïdo University, 171-188

Fukazawa K (1984) Juvenile wood of hardwoods judged by density variation. IAWA Bulletin, new series 5 , 65-73

Huber F (1991) Modélisation de l'infradensité du bois en fonction de la largeur de cerne et de l'âge médullaire chez les chênes indigènes. Annexe au compte rendu final du rapport CEE « Genetics and breeding of oaks «, $20 \mathrm{p}$

Marchal R (1983) Intérêt de la prise en compte de caractéristiques physiques et anatomiques simples du bois de chêne pour l'appréciation de la qualité des placages d'ébénisterie. DEA, Inra, Nancy, $105 \mathrm{p}$

Nepveu $G$ (1990) Les facteurs influençant la qualité du bois de chêne (chêne rouvre et chêne pédonculé). Rev For Fr 2, 128-133 
Polge $H$ (1966) Établissement des courbes de variation de la densité du bois par exploration densitométrique de radiographies d'échantillons prélevés à la tarière sur des arbres vivants. Applications dans les domaines technologique et physiologique. Thèse, université de Nancy, Nancy
Polge H, Keller R (1973) Qualité du bois et largeur d'accroissements en forêt de Tronçais. Ann Sci For 30, 91-125

Zhang SY, Eyono Owoundi R, Nepveu G, Mothe F, Dhôte JF (1993) Modelling wood density in European oak (Quercus petreae and Quercus robur) and simulating the silvicultural influence. Can J For Res 23, 2587-2593 